\title{
Deutschland hat Nachholbedarf in einigen Zukunftsindustrien
}

\begin{abstract}
Die Wettbewerbsfähigkeit der deutschen Wirtschaft steht vor gewaltigen Herausforderungen. Traditionell starke Sektoren wie die Automobilindustrie oder der Maschinenbau befinden sich angesichts disruptiver Veränderungen durch neue Technologien, den Kampf gegen den Klimawandel und veränderte regulatorische Rahmenbedingungen in einer Umbruchphase. Zahlreiche Industriezweige wandeln sich durch den Einsatz von Künstlicher Intelligenz zu „Smart Industries“. Gleichzeitig gewinnt die Kompetenz in Querschnittstechnologien wie Cloud Computing oder Cyber Security an Bedeutung, da diese den effektiven Einsatz von Künstlicher Intelligenz erst ermöglichen. Eine Analyse der Wettbewerbsposition der deutschen Wirtschaft zeigt auf, dass in manchen Zukunftsfeldern ein erheblicher Nachholbedarf besteht.
\end{abstract}

\begin{abstract}
Seit geraumer Zeit wird öffentlich darüber diskutiert, wie es um die künftige Wettbewerbsfähigkeit der deutschen Wirtschaft in Zeiten fortschreitender Digitalisierung bestellt ist. Tatsächlich befinden sich zahlreiche Industrien durch die Digitalisierung in einem radikalen Wandel. Neue Technologien, verändertes Kundenverhalten und auch der Klimawandel zwingen Wirtschaft und Gesellschaft zu einem Umdenken. Vor allem Fortschritte beim Thema "Künstliche Intelligenz" (KI) und die damit verbundenen veränderten Anforderungen an die künftige Arbeitswelt durch Industrie 4.0 sorgen bei vielen Menschen für Verunsicherung. Selbst traditionell starke Branchen wie die deutsche Automobilindustrie und der Maschinenbau stehen vor fundamentalen Veränderungen.
\end{abstract}

Im Zuge von Industrie 4.0 werden Wertschöpfungsprozesse in Produktion und Logistik innerhalb von Unternehmen und über Unternehmensgrenzen hinweg über Web-basierte Technologien miteinander vernetzt. Virtuelle und physische Systeme verschmelzen zu sogenannten Cyber-Physical Systems (CPS), sodass z. B. in der Smart Factory Produktionssysteme eigenständig Daten austauschen, Fehler erkennen und sich aufgrund von Lernprozessen selbst optimieren können. Methoden und Instrumente der Künstlichen Intel-

Prof. Dr. Volker Brühl ist Geschäftsführer am Center for Financial Studies an der Goethe-Universität in Frankfurt am Main. ligenz ermöglichen die Entstehung von Wertschöpfungsnetzwerken, die sich mit einem hohen Grad an Autonomie und Flexibilität in Echtzeit an veränderte Bedingungen (z.B. Bestellverhalten, Materialengpässe) anpassen können. Auch die Individualisierung von Produkten wird durch Industrie 4.0 erleichtert.'

Unter „Smart Industries“ werden daher nachfolgend diejenigen Branchen verstanden, deren Produkte und Dienstleistungen zunehmend intelligente Eigenschaften aufweisen werden. Diese können durch eine Interaktion mit ihrer Umwelt (z.B. über Sensoren und Aktoren) automatisiert, (teil-)autonom und selbstoptimierend ihre Aufgaben erledigen. Neben der Smart Factory sind dies beispielsweise die Bereiche Vernetzung des Haushalts (Smart Home) oder intelligente Energienetze (Smart Grid). Je nach Sektor bieten die Unternehmen „smart products“ (z. B. Smartphones) oder "smart services“ (z. B. Verkehrsleitsysteme oder digital Advertising) an. ${ }^{2}$ Es wird entscheidend darauf ankommen, dass Deutschlands Wirtschaft vor allem in zukunftsträchtigen Sektoren ihre Wettbewerbsfähigkeit hält, absichert und ausbaut. Die Bundesregierung hat diese Fragestellung unter anderem mit der Weiterentwicklung der Hightech-Strategie

(C) Der/die Autor(en) 2020. Open Access: Dieser Artikel wird unter der Creative Commons Namensnennung 4.0 International Lizenz (http:// creativecommons.org/licenses/by/4.0/deed.de) veröffentlicht.

Open Access wird durch die ZBW - Leibniz-Informationszentrum Wirtschaft gefördert.

1 Vgl. acatech/Forschungsunion: Deutschlands Zukunft als Produktionsstandort sichern - Umsetzungsempfehlungen für das Zukunftsprojekt Industrie 4.0, Berlin 2013.

2 Vgl. Plattform Industrie 4.0, Forschungsbeirat: Engineering smarter Produkte und Services Plattform Industrie 4.0, München 2018. 
2025, der Nationalen Industriestrategie 2030 sowie einer Strategie zur Künstlichen Intelligenz aufgegriffen. ${ }^{3}$

Vor diesem Hintergrund wurde untersucht, wie es um die Wettbewerbsposition der deutschen Wirtschaft in 19 Kernindustrien bestellt ist, die künftig maßgeblich durch KI geprägt sein werden oder als wichtige Querschnittstechnologien die Entwicklung dieser Sektoren befördern bzw. erst ermöglichen. Insgesamt wurden 15 „Smart Industries“ sowie vier Querschnittstechnologien (Cloud Computing, Cyber Security, Big Data/Advanced Analytics, 5G Infrastructure Technologies) untersucht, die als „enabler“ von Smart Industries eine wichtige Rolle einnehmen. ${ }^{4}$ Es sei darauf hingewiesen, dass die untersuchten Teilsektoren nur einen Ausschnitt der künftigen datengetriebenen Wachstumsmärkte abbilden können und die in Tabelle 1 aufgeführten Wachstumsraten angesichts möglicher disruptiver Marktveränderungen nur als grobe Schätzungen anzusehen sind.

\section{Smart Industries - Wachstumspotenziale und Positionierung der deutschen Wirtschaft}

Tabelle 1 gibt einen Überblick über diejenigen Wachstumsmärkte, die Gegenstand der vorliegenden Untersuchung sind. Es zeigt sich, dass die Bandbreite der von KI geprägten Smart Industries sehr groß ist. Betroffen sind Branchen wie der Maschinenbau, der durch intelligente Automatisierungstechnologien und Robotik revolutioniert wird (Smart Factory), und der Mobilitätssektor (intelligente Verkehrssysteme, innovative Mobilitätsdienstleistungen oder (teil-)autonomes Fahren). Aber auch intelligente Stromnetze (Smart Grids), die Vernetzung von Gebäuden (Smart Building) und Haushalten (Smart Home) sind hier zu nennen. Darüber hinaus werden mit den Bereichen Smartphones/Tablets, Smart TV, Wearables und Gaming wesentliche Teile der Unterhaltungselektronik (Consumer Electronics) einbezogen. Ergänzend fließen die nach wie vor wachsenden Bereiche E-Commerce und Digital Advertising ebenso ein wie die noch kleinen, aber mit hohem Wachstumspotenzial verbundenen Märkte für Service-Roboter und Smart Farming.

In Tabelle 1 werden je Sektor die wichtigsten Produkte/ Dienstleistungen, das gegenwärtige Marktvolumen sowie

3 Vgl. Bundesministerium für Wirtschaft und Energie: Nationale Industriestrategie 2030, Strategische Leitlinien für eine deutsche und europäische Industriepolitik, Berlin, Februar 2019; Bundesministerium für Bildung und Forschung: Die Hightech-Strategie 2025, Berlin, August 2018; Die Bundesregierung: Strategie Künstliche Intelligenz der Bundesregierung, Berlin, November 2018.

4 Die Blockchain-Technologie wurde in dieser Analyse nicht berücksichtigt. Einen Überblick bietet z.B. V. Brühl: Bitcoins, Blockchain und Distributed Ledgers, in: Wirtschaftsdienst, 97. Jg. (2017), H. 2, S. 135-142, https://www.wirtschaftsdienst.eu/inhalt/jahr/2017/heft/2/ beitrag/bitcoins-blockchain-und-distributed-ledgers-funktionsweise-marktentwicklungen-und-zukunftsperspektiv.html (7.2.2020). die erwarteten durchschnittlichen Wachstumsraten bis zum Jahr 2028 aufgeführt. Bei der Abschätzung von Wachstumsraten in den betrachteten Märkten ist zu berücksichtigen, dass aufgrund ihrer noch jungen Historie nur im begrenzten Umfang auf historische Daten zurückgegriffen werden kann. Daher beruhen die Wachstumsprognosen in der Regel auf den Untersuchungen spezialisierter Marktforschungsinstitute, die zum einen auf Befragungen von Marktteilnehmern und Industrie-Experten beruhen (Bottom-up) und zum anderen mit makroökonomischen Wachstumsprognosen (Topdown) abgeglichen werden. Es wurden je nach Verfügbarkeit unterschiedliche Marktstudien je Sektor analysiert und gegebenenfalls eine Konsensus-Schätzung als Durchschnittswert abgeleitet.

Außerdem werden eine Auswahl der global wichtigen Anbieter sowie der wesentlichen deutschen Unternehmen in dem jeweiligen Sektor gezeigt, wobei je nach (Teil-)Industrie auch solche Unternehmen einbezogen werden, die nicht branchenweit, sondern gegebenenfalls nur in gewissen Segmenten der jeweiligen Industrie eine marktführende Position einnehmen. ${ }^{5}$ Aus der relativen Bedeutung der deutschen Anbieter für den jeweiligen globalen Sektor wird dann die Wettbewerbsposition der deutschen Wirtschaft in drei Kategorien eingeteilt und in der Tabelle durch einen vollständig ausgefüllten Kreis (starke Position), einen halb gefüllten Kreis (durchschnittliche Position) bzw. durch einen leeren Kreis (schwache Position) symbolisiert. Von einer starken Wettbewerbsposition der deutschen Wirtschaft wird dann ausgegangen, wenn in dem betreffenden Sektor sowohl multinationale Konzerne mit Hauptsitz in Deutschland zu den führenden Unternehmen gehören als auch mehrere mittelständische deutsche Unternehmen eine führende Marktposition in bestimmten Marktsegmenten des betreffenden Sektors einnehmen.

Entsprechend wird die Wettbewerbsposition der deutschen Wirtschaft dann als durchschnittlich in einem Sektor charakterisiert, wenn deutsche Unternehmen in diesem nur punktuell, sei es durch einzelne Konzerne oder Mittelständler, in ausgewählten Segmenten vertreten sind. In solchen Sektoren decken deutsche Unternehmen nicht das volle industrielle Leistungsspektrum im jeweiligen Sektor ab, etwa weil es an technologischen Kompetenzen, einer entwickelten Zulieferindustrie oder einem Zugang zu den Endkundenmärkten fehlt. Eine schwache Positionierung der deutschen Wirtschaft wird nachfolgend dann konstatiert, wenn in dem jeweiligen Sektor nur wenige, nicht marktführende deutsche Anbieter zu verzeichnen sind bzw. deutsche Anbieter kom-

5 Nachfolgend werden diejenigen Firmen als „deutsche Unternehmen“ bezeichnet, die ihren Hauptsitz in Deutschland haben, da in diesem Fall unterstellt wird, dass die wesentlichen strategischen Entscheidungen in Deutschland getroffen werden. 
Tabelle 1

Marktpotenziale und deutsche Wettbewerbsposition in „Smart Industries“ und ausgewählten Querschnittstechnologien

\begin{tabular}{|c|c|c|c|c|c|c|c|}
\hline Sektor & Produkte/Services & $\begin{array}{l}\text { Markt- } \\
\text { volumen } \\
2018 \\
\text { (Mrd. } \\
\text { US-\$) }\end{array}$ & $\begin{array}{l}\text { Globales } \\
\text { Markt- } \\
\text { potenzial } \\
2028 \text { (Mrd. } \\
\text { US-\$) }\end{array}$ & $\begin{array}{l}\text { Wachs- } \\
\text { tum }^{1} \\
(\%)\end{array}$ & $\begin{array}{l}\text { Marktführende Anbieter } \\
\text { (global, Auswahl, ohne deutsche } \\
\text { Anbieter) }\end{array}$ & $\begin{array}{l}\text { Deutsche } \\
\text { Anbieter } \\
\text { (Auswahl) }\end{array}$ & $\begin{array}{l}\text { Wettbe- } \\
\text { werbspo- } \\
\text { sition der } \\
\text { deutschen } \\
\text { Wirtschaft }\end{array}$ \\
\hline $\begin{array}{l}\text { Smart Fac- } \\
\text { tory }\end{array}$ & $\begin{array}{l}\text { Sensors, Industrial Robots, Ma- } \\
\text { chine Vision Systems, industrial } \\
\text { 3D printing, prducts/compo- } \\
\text { nents: DCS, PLC, MES, ERP, } \\
\text { SCADA, PAM, HMI, PLM }\end{array}$ & 140 & 280 & 7,2 & $\begin{array}{l}\text { ABB (CH), Atos (FR), Emerson Electric } \\
\text { (US), FANUC (JP), GE (US), Honeywell } \\
\text { (US), Kuka (CN), Schneider (FR), 3D } \\
\text { Systems (US), AddUP (FR) }\end{array}$ & $\begin{array}{l}\text { Bosch, Sie- } \\
\text { mens, EOS, } \\
\text { SLM, Trumpf, } \\
\text { Festo }\end{array}$ & \\
\hline Smart Grid & $\begin{array}{l}\text { Intelligent Energy Management } \\
\text { Systems (generation, distribu- } \\
\text { tion, consumption), Advanced } \\
\text { Metering (AMI), Billing, Custo- } \\
\text { mer Information Systems }\end{array}$ & 24 & 90 & 14,2 & $\begin{array}{l}\text { General Electric (US), ABB (CH), } \\
\text { Itron (US), Cisco (US), NexGrid (US), } \\
\text { Landis+Gyr (CH), Wipro (IN), Cooper- } \\
\text { PowerSystems (US), Panasonic (JP), } \\
\text { Hitachi (JP), Honeywell (US), Mitsubi- } \\
\text { shi (JP), Sagemcom (FR) }\end{array}$ & $\begin{array}{l}\text { Siemens, SAP, } \\
\text { Deutsche } \\
\text { Telekom, Diehl, } \\
\text { PSI, PPC, } \\
\text { verschiedene } \\
\text { Utilities }\end{array}$ & \\
\hline $\begin{array}{l}\text { Intelligent } \\
\text { Traffic Sys- } \\
\text { tems }\end{array}$ & $\begin{array}{l}\text { Advanced Traffic Management } \\
\text { Systems, Advanced Traveler } \\
\text { Information Systems, ITS- } \\
\text { Enabled Transportation Pricing } \\
\text { Systems }\end{array}$ & 23 & 40 & 5,5 & $\begin{array}{l}\text { Thales (FR), Garmin (CH), Kapsch } \\
\text { (AT), TomTom (NL), EFKON (AT), FLIR } \\
\text { (US), Denso (JP), Iteris (US), Ricardo } \\
\text { (UK), Savari (US), Transcore (US), } \\
\text { Swarco (AT) }\end{array}$ & $\begin{array}{l}\text { Siemens, T- } \\
\text { Systems, SAP, } \\
\text { CargoSoft, PSI }\end{array}$ & \\
\hline $\begin{array}{l}\text { Intelligent } \\
\text { Buildings }\end{array}$ & $\begin{array}{l}\text { Infrastructure Management } \\
\text { Systems (z. B. Energy, IT, } \\
\text { Security) }\end{array}$ & 9 & 70 & 22,4 & $\begin{array}{l}\text { ABB (CH), Cisco (US), Schneider } \\
\text { Electric (FR), Johnson Controls (US), } \\
\text { United Technologies (US), Emerson } \\
\text { Electric (US) }\end{array}$ & $\begin{array}{l}\text { Siemens, euro- } \\
\text { micron }\end{array}$ & \\
\hline $\begin{array}{l}\text { Connected } \\
\text { Car Solu- } \\
\text { tions }\end{array}$ & $\begin{array}{l}\text { Autonomous Driving, V2V (Ve- } \\
\text { hicle to Vehicle), V2I (Vehicle to } \\
\text { Infrastructure), V2C (Vehicle to } \\
\text { Cloud), Advanced Driver Assis- } \\
\text { tance Systems, On-Board Unit } \\
\text { (OBU), Roadside Unit (RSU), } \\
\text { Smart Antennas, Displays, } \\
\text { Electronic Control Unit, } \\
\text { Sensors }\end{array}$ & 69 & 280 & 15,0 & $\begin{array}{l}\text { Denso (JP), Delphi (UK), Harman (US), } \\
\text { Autoliv (SE), Aisin Seiki (JP), Visteon } \\
\text { (US), Google (US), verschiedene Auto- } \\
\text { hersteller }\end{array}$ & $\begin{array}{l}\text { Bosch, } \\
\text { Continental, } \\
\text { verschiedene } \\
\text { Autohersteller }\end{array}$ & \\
\hline $\begin{array}{l}\text { Ride Hailing/ } \\
\text { Carsharing }\end{array}$ & $\begin{array}{l}\text { E-hailing, Station-Based, } \\
\text { Carsharing \& Rental }\end{array}$ & 73 & 450 & 19,9 & $\begin{array}{l}\text { Uber (US), Lyft (US), DiDi (CN), Grab } \\
\text { (Singapore), Gett (IL), Ola (IN), BlaBla- } \\
\text { Car (FR), Lime (US), Hertz (US), Zipcar } \\
\text { (US), Orix (JP) }\end{array}$ & $\begin{array}{l}\text { Car2go, } \\
\text { DriveNow }\end{array}$ & \\
\hline $\begin{array}{l}\text { Smart } \\
\text { Phones }\end{array}$ & Android, iOS, other & 522 & 580 & 1,1 & $\begin{array}{l}\text { Samsung (KR), Apple (US), Huawei } \\
(\mathrm{CN}) \text {, Xiaomi (CN), Oppo (CN), HTC } \\
\text { (TW), Sony (JP), LG (KR), Nokia (FI), } \\
\text { ZTE (CN) }\end{array}$ & $\begin{array}{l}\text { kein (führender) } \\
\text { Anbieter }\end{array}$ & \\
\hline Gaming & Console, mobile, PC & 139 & 330 & 9,1 & $\begin{array}{l}\text { Sony (JP), Microsoft (US), Electronic } \\
\text { Arts (US), Nintendo (JP), Tencent } \\
\text { Games (CN), Ubisoft (US), Activision } \\
\text { Blizzard (CN), NewZoo (NL) }\end{array}$ & $\begin{array}{l}\text { Crytek, BlueBy- } \\
\text { te, InnoGames, } \\
\text { versch. kleinere } \\
\text { Anbieter }\end{array}$ & \\
\hline Smart Home & $\begin{array}{l}\text { Lighting Control, Security \& Ac- } \\
\text { cess Control, HVAC, Entertain- } \\
\text { ment, Smart Speaker, Home } \\
\text { Healthcare, Home Appliances, } \\
\text { Smart Furniture }\end{array}$ & 77 & 180 & 8,9 & $\begin{array}{l}\text { Johnson Controls (US), United } \\
\text { Technologies (US), Honeywell (US), } \\
\text { Amazon (US), Apple (US), Google } \\
\text { (US), Legrand (FR), GE (US), Samsung } \\
\text { (KR), LG Electronics (KR), Sony (JP) }\end{array}$ & $\begin{array}{l}\text { Siemens, } \\
\text { Bosch, } \\
\text { RWE }\end{array}$ & \\
\hline Smart TV & $\begin{array}{l}\text { 4K, UHD TV, HD TV, full HD TV, } \\
8 \mathrm{~K} T V \text {, platforms (Android, iOS, } \\
\text { Roku, Web OS, Tizen OS), tech- } \\
\text { nology (z. B. OLED, QLED) }\end{array}$ & 157 & 320 & 7,4 & $\begin{array}{l}\text { LG Electronics (KR), Samsung (KR), } \\
\text { Sony (JP), Apple (US), Philips (NL), } \\
\text { TCL (CN), Haier Group Corporation } \\
\text { (CN), Hitachi (JP) }\end{array}$ & $\begin{array}{l}\text { kein (führender) } \\
\text { Anbieter }\end{array}$ & \\
\hline
\end{tabular}

- starke Marktposition; 0 durchschnittliche Marktposition; O schwache Marktposition.

${ }^{1}$ Wachstumsrate p.a. 2018-2028.

Quellen: International Data Corporation IDC, International Federation of Robotics IFR, Gesellschaft für Konsumforschung GfK, MarketsandMarkets, eMarketer, Mordor Intelligence, Research and Markets, Android Market Research, NewZoo.com, Juniper Research, Expert Interviews, eigene Berechnungen. 
Tabelle 1 (Fortsetzung)

Marktpotenziale und deutsche Wettbewerbsposition in „Smart Industries“ und ausgewählten Querschnittstechnologien

\begin{tabular}{|c|c|c|c|c|c|c|c|}
\hline Sektor & Produkte/Services & $\begin{array}{l}\text { Markt- } \\
\text { volumen } \\
2018 \\
\text { (Mrd. } \\
\text { US-\$) }\end{array}$ & $\begin{array}{l}\text { Globales } \\
\text { Markt- } \\
\text { potenzial } \\
2028 \\
\text { (Mrd. } \\
\text { US-\$) }\end{array}$ & $\begin{array}{l}\text { Wachs- } \\
\text { tum }^{1} \\
(\%)\end{array}$ & $\begin{array}{l}\text { Marktführende Anbieter } \\
\text { (global, Auswahl, ohne deutsche } \\
\text { Anbieter) }\end{array}$ & $\begin{array}{l}\text { Deutsche } \\
\text { Anbieter } \\
\text { (Auswahl) }\end{array}$ & $\begin{array}{l}\text { Wettbe- } \\
\text { werbspo- } \\
\text { sition der } \\
\text { deutschen } \\
\text { Wirtschaft }\end{array}$ \\
\hline Wearables & $\begin{array}{l}\text { Smart Watches, Fitness Tra- } \\
\text { cker, medical devises, AR/VR }\end{array}$ & 27 & 80 & 11,5 & $\begin{array}{l}\text { Fitbit (US), Apple (US), Xiaomi (CN), } \\
\text { Garmin (US), Samsung (KR), Alphabet } \\
\text { (US), LG (KR), Sony (JP), Jawbone } \\
\text { (US), Misfit (US), Lifesense (NL) }\end{array}$ & $\begin{array}{l}\text { kein (führender) } \\
\text { Anbieter }\end{array}$ & \\
\hline $\begin{array}{l}\text { Service } \\
\text { Robotics }\end{array}$ & $\begin{array}{l}\text { service robotics for private } \\
\text { households (e.g. cleaning), } \\
\text { professional service robotics } \\
\text { (z. B. logistics, medical care, } \\
\text { security) }\end{array}$ & 14 & 150 & 27,2 & $\begin{array}{l}\text { iRobot (US), amazon robotics (US), } \\
\text { Yaskawa (JP), LG (KR), Honda (JP), } \\
\text { Husqvarna (SE), Samsung (KR), } \\
\text { OmronAdept Technologies (US), Kuka } \\
\text { (CN), Intuitive Surgical (US), Stryker } \\
\text { (US), Smith \& Nephew (US) }\end{array}$ & $\begin{array}{l}\text { kein (führender) } \\
\text { Anbieter }\end{array}$ & \\
\hline E-commerce & Retail (B2C) & 2928 & 10000 & 13,1 & $\begin{array}{l}\text { Amazon (US), Alibaba (CN), eBay } \\
\text { (US), Jingdong (CN), Rakuten (JP), } \\
\text { Homedepot (US), Flipkart (IN) }\end{array}$ & Otto, Zalando & \\
\hline $\begin{array}{l}\text { Digital } \\
\text { Advertising }\end{array}$ & $\begin{array}{l}\text { Display marketing (banner, } \\
\text { pop-ups), SEO, Social Media } \\
\text { Marketing, Affiliate Marketing }\end{array}$ & 255 & 700 & 10,6 & $\begin{array}{l}\text { Google (US), Facebook (US), Alibaba } \\
\text { (CN), Amazon (US), Baidu (CN), } \\
\text { Tencent (CN), Microsoft (US), Verizon } \\
\text { (US), Twitter (US) }\end{array}$ & $\begin{array}{l}\text { kein (führender) } \\
\text { Anbieter }\end{array}$ & \\
\hline $\begin{array}{l}\text { Smart } \\
\text { Farming }\end{array}$ & $\begin{array}{l}\text { Precision Farming Applica- } \\
\text { tions, Livestock Monitoring } \\
\text { Applications, Automation and } \\
\text { Control Systems, Sensing and } \\
\text { Monitoring Systems }\end{array}$ & 8 & 30 & 14,7 & $\begin{array}{l}\text { Deere (US), Trimble (US), AGCO (US), } \\
\text { Fiat (IT), DeLaval (SE), Precision Plan- } \\
\text { ting (US), CropMetrics (US), Agribotix } \\
\text { (US), Descartes Labs (US), Gamaya } \\
\text { (CH), Granular (US) }\end{array}$ & Claas, GEA & \\
\hline $\begin{array}{l}\text { Cloud } \\
\text { Computing }\end{array}$ & $\begin{array}{l}\text { Infrastructure as a Service } \\
\text { (laaS), Platform as a Service } \\
\text { (PaaS), Software as a Service } \\
\text { (SaaS) }\end{array}$ & 182 & 840 & 16,5 & $\begin{array}{l}\text { AWS (US), Microsoft (US), Google } \\
\text { (US), Alibaba (China), IBM (US), Oracle } \\
\text { (US), VMware (US), Rackspace (US), } \\
\text { Salesforce (US), Verizon (US), Centu- } \\
\text { ryLink (US), Fujitsu (JP), NTT (JP) }\end{array}$ & SAP & \\
\hline $\begin{array}{l}\text { Cyber } \\
\text { Security } \\
\text { Market }\end{array}$ & $\begin{array}{l}\text { Firewall, Antivirus/Antimalware, } \\
\text { Identity and Access Manage- } \\
\text { ment (IAM), Data Loss Preven- } \\
\text { tion (DLP), Intrusion Detection } \\
\text { System (IDS)/Intrusion Preven- } \\
\text { tion System (IPS), Distributed } \\
\text { denial of service (DDoS) }\end{array}$ & 125 & 400 & 12,3 & $\begin{array}{l}\text { IBM (US), Check Point (US), CyberArk } \\
\text { (IL), F5 Networks (US), Amazon Web } \\
\text { Services (US), Oracle (US), McAfee } \\
\text { (US), Micro Focus (UK), Microsoft } \\
\text { (US), Sophos (UK), Splunk (US), Sym- } \\
\text { antec (US), Trend Micro (JP) }\end{array}$ & $\begin{array}{l}\text { kein (führender) } \\
\text { Anbieter }\end{array}$ & \\
\hline $\begin{array}{l}\text { Big Data/ } \\
\text { Advanced } \\
\text { Analytics }\end{array}$ & $\begin{array}{l}\text { Big Data, Advanced Analytics } \\
\text { solutions incl. analysis, que- } \\
\text { rying, visualization, and dash- } \\
\text { boarding tools, data warehouse } \\
\text { management tools, cognitive/Al } \\
\text { software platforms }\end{array}$ & 169 & 550 & 12,5 & $\begin{array}{l}\text { Information Builders (US), Microsoft } \\
\text { (US), Salesforce (US), Sisense (US), } \\
\text { Oracle (US), GoodData (US), IBM } \\
\text { (US), Logi Analytics (US), MicroStrat- } \\
\text { egy (US), SAS (US), UEC (CN), China } \\
\text { Cache (CN) }\end{array}$ & SAP & \\
\hline $\begin{array}{l}\text { Mobile Infra- } \\
\text { structure } \\
(5 G)\end{array}$ & $\begin{array}{l}\text { 5G RAN, } 5 \text { G Core, small cell, } \\
\text { macro cell, Network Functions } \\
\text { Virtualisation Infrastructure } \\
\text { (NFVI) }\end{array}$ & 1 & 30 & 43,9 & $\begin{array}{l}\text { Huawei (CN), Ericsson (SE), Samsung } \\
\text { (KR), Nokia (FL), ZTE (CN), NEC (JP), } \\
\text { Cisco (US) }\end{array}$ & $\begin{array}{l}\text { kein (führender) } \\
\text { Anbieter }\end{array}$ & \\
\hline Insgesamt & & 4941 & 15400 & 12,0 & & & \\
\hline
\end{tabular}

- starke Marktposition; O durchschnittliche Marktposition; O schwache Marktposition.

${ }^{1}$ Wachstumsrate p.a. 2018-2028.

Quellen: International Data Corporation IDC, International Federation of Robotics IFR, Gesellschaft für Konsumforschung GfK, MarketsandMarkets, eMarketer, Mordor Intelligence, Research and Markets, Androit Market Research, NewZoo.com, Juniper Research, Expert Interviews, eigene Berechnungen. 
plett fehlen. Die Übersicht in Tabelle 1 verdeutlicht, dass sich das globale Marktpotenzial von „Smart Industries“ bzw. den dazu gehörigen Kerntechnologien im Zeitraum von 2018 bis 2028 verdreifachen und 2028 bei mehr als 15 Billionen US-\$ liegen dürfte. Die erwarteten Wachstumsraten reichen von $1 \%$ im sich einer Sättigungsgrenze nähernden Markt für Smartphones bis zu ca. $43 \%$ p. a. im Bereich der mobilen Telekommunikationsinfrastruktur durch die Einführung der 5G-Technologie.

\section{Deutschland mit starker Wettbewerbsposition}

Wenn man die unterschiedlichen Segmente betrachtet, gibt es einige Felder, in denen Deutschland sehr gut positioniert ist. Dazu zählt der Bereich „Smart Factory“. Deutsche Unternehmen wie Siemens, Bosch, Trumpf oder EOS sind hier neben zahlreichen mittelständischen Unternehmen als Innovationsführer vertreten. Im Bereich der Industrie-Roboter hat Deutschland seit der Übernahme von Kuka durch einen chinesischen Investor keine nennenswerte Präsenz mehr. Das globale Marktpotenzial dürfte sich im Bereich Smart Factory in den kommenden zehn Jahren auf etwa 280 Mrd. US-\$ verdoppeln. Ähnlich stark vertreten ist die deutsche Wirtschaft im Zukunftsfeld „Connected-Car-Services“, zu dem unter anderem das (teil-)autonome Fahren sowie intelligente Fahrerassistenzsysteme gehören. Neben Firmen wie Siemens, Bosch und Continental sind im Bereich des (teil-)autonomen Fahrens auch deutsche Autohersteller gut vertreten, die sich aber harter internationaler Konkurrenz und dem Markteintritt neuer Anbieter wie Google gegenübersehen. Auch hier nehmen zahlreiche deutsche mittelständische Unternehmen führende Positionen bei bestimmten Komponenten (z.B. Sensoren) ein. Dieser Befund ist aufgrund der traditionellen Stärken Deutschlands im Maschinen- und Anlagenbau bzw. der Automobilindustrie nicht überraschend. Allerdings sind erhebliche Anstrengungen erforderlich, diese beschäftigungsintensiven Sektoren mit einem möglichst hohen Wertschöpfungsanteil in Deutschland zu halten.

Denn in den Bereichen Elektromobilität und innovative Antriebstechnologien tun sich deutsche Hersteller im Vergleich zur ausländischen Konkurrenz noch schwer. Der Bereich der Elektromobilität im engeren Sinne ist nicht Gegenstand dieser Untersuchung, da dessen Wachstum nicht primär von Künstlicher Intelligenz, sondern der Weiterentwicklung auf den Gebieten der Ladeinfrastruktur, Batterietechnologien oder E-Motoren abhängt. Sehr gut aufgestellt sind deutsche Unternehmen auch in den Bereichen Smart Grid (Intelligente Stromnetze) und intelligente Mobilitätssteuerungssysteme. Smart-Grid-Lösungen sind unverzichtbarer Bestandteil der Energiewende, da der Umstieg auf erneuerbare Energien ein flexibles, intelligentes Management von Produktion, Verteilung und Nutzung von Energie voraussetzt. Hier ist Deutschland mit Siemens, einigen Energieerzeugern oder Übertra- gungsnetzbetreibern und manchen Mittelständlern (z.B. Diehl, PSI) durchaus gut vertreten, allerdings ist der Fokus bis auf wenige Ausnahmen stark auf den nationalen Energiemarkt ausgerichtet.

\section{Ausbaufähige Positionen}

Daneben gibt es einige Bereiche, in denen deutsche Unternehmen zwar vereinzelt, aber nicht in der industriellen Breite gut aufgestellt sind. Dies gilt beispielsweise für die Sektoren „Smart Farming“ (intelligente Landwirtschaft), „Smart Home“ (intelligente Vernetzung zu Hause) oder "innovative Mobilitätsdienstleistungen“, zu denen unter anderem Carsharing, Ride Hailing oder Ride Sharing gehören. Im klassischen Carsharing haben die beiden deutschen großen Anbieter Car2Go und DriveNow durch ihren Zusammenschluss zu ShareNow ihre Kapazitäten gebündelt. In den Bereichen Ride Hailing oder Ride Sharing wird durch die App-basierte Vermittlung privater Fahrdienste ein enormes Wachstumspotenzial erwartet. Neben den US-Konzernen Uber und Lyft haben inzwischen auch deutsche Anbieter wie z. B. Moia (VW-Konzern) oder ViaVan (Daimler) mit ähnlichen Geschäftsmodellen den Markteintritt vollzogen, sind aber im internationalen Vergleich noch sehr klein. Gerade in diesen Feldern wird es darauf ankommen, die regulatorischen Voraussetzungen und die damit verbundene Planungssicherheit zu schaffen, damit sich innovative Mobilitätsdienstleistungen auch hierzulande rasch etablieren können. Auch bei der Digitalisierung der Landwirtschaft spielen deutsche Unternehmen eine untergeordnete Rolle, obwohl der Einsatz von $\mathrm{KI}$ als ein wichtiger Baustein bei der Bekämpfung des Klimawandels und von Hungersnöten gesehen wird.

Unter dem Begriff „Smart Home“ subsumiert man unterschiedliche Anwendungsfelder, wie vernetzte Haushaltsgeräte, Sicherheitssysteme, intelligente Beleuchtungs- oder Heizungssysteme sowie intelligente Sprachassistenten (smart speaker). Dieser Markt wird von zahlreichen internationalen Anbietern (z. B. Johnson Controls, United Technologies, Schneider Electric, Honeywell, Apple, Amazon, LG oder Samsung) bearbeitet. Deutsche Anbieter wie Bosch, Siemens oder Miele sind hier ebenfalls substanziell vertreten, allerdings vor allem in den Premiumsegmenten von Haushaltsgeräten und weniger bei der intelligenten Steuerung über Sprachassistenten.

\section{Deutschland in einigen Bereichen kaum vertreten}

In einigen $\mathrm{Kl}$-getriebenen Wachstumsmärkten ist Deutschland so gut wie gar nicht vertreten. Deutsche Unternehmen sind aus den Märkten für Consumer Electronics (Smartphones, Smart TV) nahezu verschwunden oder wie in den schnell wachsenden Märkten für Wearables kaum vertreten. Wearables sind kleine vernetzte Computer, die am Körper 
des Nutzers getragen werden und den Alltag des Nutzers unterstützen sollen. Bekannte Beispiele sind Smart Watches, Fitnessarmbänder und digitale Brillen. Wearables finden jedoch auch zunehmend im medizinischen Bereich Anwendung. Dazu zählen z.B. digitale Blutzucker- und Blutdruck-Messgeräte. Deutsche Hersteller sind hier kaum zu finden. Der Bereich der Service-Roboter, die in Haus und Garten, Pflege oder Sicherheit eingesetzt werden, wächst ebenfalls mit zweistelligen Wachstumsraten. Auch dieser Markt wird von ausländischen Anbietern wie z.B. iRobot, amazon robotics (USA), Yaskawa, Honda (beide Japan), LG, Samsung (Korea) oder Husqvarna (Schweden) beherrscht. Nicht zu vergessen ist auch die Dominanz US-amerikanischer und chinesischer Konzerne im Bereich E-Commerce (Amazon, Alibaba), deren Abstand zu den übrigen Marktteilnehmern immer größer zu werden scheint. Erfreulich ist, dass Deutschland mit Zalando ein Unternehmen hervorgebracht hat, das neben der Otto Group zumindest in seinen Segmenten beachtliche Erfolge erzielt. Ähnlich ist die Situation im digital Advertising, in denen die US-Tech-Giganten uneinholbar vorne liegen.

Oft vernachlässigt wird auch der stark wachsende Bereich des Gaming, zu dem Spielkonsolen, Smartphone- oder PCgestützte Spiele und auch der Bereich E-Sports gehören. Auch dieser Markt wird von US-amerikanischen (Microsoft, Electronic Arts, Activision Blizzard), japanischen (Sony, Nintendo) und chinesischen Akteuren (Tencent Games) dominiert. Dabei wird dieser Markt voraussichtlich bis zum Jahr 2028 mit einem geschätzten Marktvolumen von 330 Mrd. US-\$ größer sein als der Markt für (Smart)TV. In Deutschland gibt es eine Reihe kleinerer Anbieter wie Crytek oder BlueByte, die aber international keine Rolle spielen.

\section{Nachholbedarf in Querschnittstechnologien}

Alarmierend ist die schwache Position deutscher Unternehmen in wichtigen Querschnittstechnologien wie den Bereichen Cyber Security, Cloud Computing oder Big-Data-/ Advanced-Analytics-Lösungen, die sektorübergreifend eine wichtige Rolle einnehmen. Die international führenden Anbieter von Cloud-Computing-Lösungen kommen überwiegend aus den USA (Amazon, Google, IBM, Oracle,
Microsoft), aus China (Alibaba) oder Japan (Fujitsu, NTT). Auch im Bereich Big Data/Advanced Analytics beherrschen US-Unternehmen den Markt. Auffällig ist, dass neben den bekannten Tech-Konzernen eine Reihe von mittelgroßen Anbietern wie Information Builders, Sisense, SAS oder Microstrategy über eine starke Marktposition in bestimmen Applikationen verfügt. Im kritischen Bereich der Cyber Security fehlt es gänzlich an international wettbewerbsfähigen deutschen Unternehmen. Dies gilt auch für kritische Infrastrukturen z. B. im Bereich der Ausrüstung von 5G-Netzen. Auch in diesem Markt gibt es seit dem Rückzug von Siemens keinen deutschen Anbieter mehr.

Es gibt Anlass zur Sorge, wenn deutsche Unternehmen in entscheidenden Zukunftsfeldern bis auf wenige Ausnahmen (z.B. SAP) nicht vertreten sind, obwohl die deutsche Forschung z. B. in den Bereichen Künstliche Intelligenz oder Cyber Security international eine Spitzenposition einnimmt. Offenbar mangelt es trotz vielfältiger Bemühungen nach wie vor in weiten Teilen an einem raschen Transfer angewandter Forschungsergebnisse in marktfähige Produkte. Hinzu kommt, dass trotz erkennbarer Fortschritte bei innovativen Start-ups in Deutschland im internationalen Vergleich immer noch eine Knappheit an Risikokapital besteht. Erfolgreiche Unternehmensgründungen wie N26 oder Delivery Hero, die den Status eines Unicorns und damit einen Marktwert von mindestens 1 Mrd. US-\$ erreicht haben, sind hierzulande immer noch eine Ausnahme.

Zusammenfassend ist festzuhalten, dass Deutschland trotz der starken Positionierung in manchen Bereichen die Gefahr droht, in wichtigen Zukunftsmärkten den Anschluss zu verlieren. Hier müssen Wirtschaft, Politik und Wissenschaft gemeinsam Konzepte entwickeln, um bestehende bürokratische Hemmnisse abzubauen, deutlich mehr Finanzmittel für die Forschung zu mobilisieren und europäische Netzwerke zu etablieren. Bereits am 7. Juni 2018 hat die Europäische Kommission einen Vorschlag für das künftige Rahmenprogramm für Forschung und Innovation (Horizont Europa) mit einem geplanten Budget von ca. 94 Mrd. Euro vorgelegt. Das Rahmenprogramm sollte zeitnah verabschiedet werden. Es könnte ein wichtiger Baustein zur Stärkung der europäischen Wirtschaft in wichtigen Zukunftsfeldern sein.

Title: Germany Has Some Catching Up to Do in Certain Future Industries

Abstract: The German economy is faced with tremendous challenges in order to remain competitive. Traditionally strong sectors such as the automotive industry and mechanical engineering are in a phase of upheaval due to new, disruptive technologies, the fight against climate change and revised regulatory frameworks. The use of artificial intelligence (Al) is transforming many industrial sectors into "smart industries". At the same time, competence in cross-sectional technologies such as cloud computing and cyber security is gaining in importance, as these are crucial for the effective use of Al. This article analyses the competitive position of the German economy in 15 future sectors and 4 cross-sectional technologies that could see their combined market potential rise to over USD 15 trillion over the next 10 years. The evidence indicates that the German economy has a lot of catching up to do in certain forward-looking sectors. JEL Classification: $A 1,03, L 6$ 